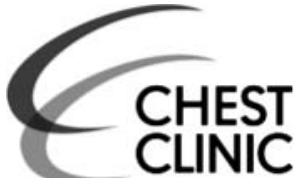

1Department of Radiology, St George's Hospital, London, UK

${ }^{2}$ Department of Respiratory Medicine, St George's Hospital, London, UK

${ }^{3}$ Department of Histopathology, St George's Hospital, London, UK

${ }^{4}$ Department of Radiology, Royal Brompton Hospital, London, UK

\section{Correspondence to} Dr Praveen Pissay Gopala Rao, Department of Radiology, St George's Hospital, Blackshaw Road, Tooting London SW17 0QT, UK; drpgpraveen@gmail.com

Received 16 February 2012 Accepted 26 May 2012 Published Online First 6 July 2012

\title{
Unexplained dyspnoea in a patient with idiopathic myelofibrosis
}

\author{
Praveen Pissay Gopala Rao, ${ }^{1}$ Rachel Buxton-Thomas, ${ }^{2}$ Brendan Tinwell, ${ }^{3}$ \\ Adrian Draper, ${ }^{2}$ Johnny Vlahos, ${ }^{1}$ Simon L F Walsh ${ }^{4}$
}

A 41-year-old with idiopathic myelofibrosis was referred to the chest clinic with a 1-month history of progressive dyspnoea and intermittent wheeze. There was no history of haemoptysis and the patient was a lifelong non-smoker. On examination, the patient was afebrile, cachectic and had hepatosplenomegaly. Haemoglobin was $6.0 \mathrm{~g} / \mathrm{dl}$, white cell count $3.6 \times 10^{9} / 1$, platelet count $98 \times 10^{9} / 1$. Blood smear showed marked anisopoikilocytes, prominent tear drop cells and polychromasia. Bone marrow aspirate was hypercellular, showed reduction in the degree of maturation, and a marked decrease in the proportion of erythroid cells. There was no evidence of acute leukaemia or metastatic malignancy. Microbiologic analysis of the sputum and blood for pulmonary infection was negative. Lung function test showed a restrictive pattern and a DLco of $32 \%$ predicted. Chest radiography demonstrated diffuse bilateral reticular interstitial infiltrates and bibasal septal lines. A subsequent CT of the chest demonstrated diffuse bilateral smooth and nodular interlobular septal thickening with centrilobular nodules (figure 1). There was no echocardiographic or radiological evidence of pulmonary hypertension.

On the basis of the patient's unexplained progressive dyspnoea, impaired gas transfer and CT findings, an open VATS-assisted lung biopsy was performed. Histology demonstrated pulmonary congestion and haemorrhage (figure 2) and numerous dark-staining hyperchromatic cells with multilobated nuclei within alveolar capillaries. On CD42B immunostain and high power (inset figure 3), these

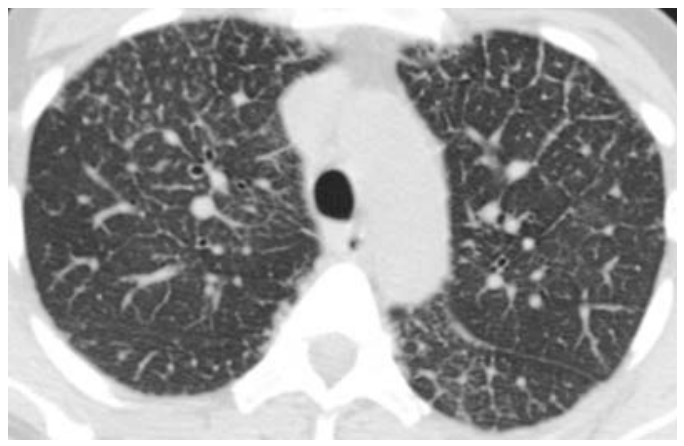

Figure $1 \mathrm{CT}$ : Representative image on lung windows through the upper lobes demonstrates diffuse nodular interlobular septal thickening which clearly defines a secondary pulmonary lobule. Small centrilobular nodules are also evident.

\section{Learning points}

Pulmonary EMH is an uncommon and typically late manifestation of myelofibrosis.

Unexplained dyspnoea, diffuse interstitial infiltrates on chest radiograph, and diffuse interlobular septal thickening on CT in patients with haematological malignancies should prompt the clinician to consider pulmonary EMH.

cells were confirmed as megakaryocytes (figures 2 and 3). The absence of CD34 expression (inset figure 2) in the peribronchiolar immature myeloid precursor cells and lymphoid cells excluded leukaemic infiltration. There was no evidence of interstitial fibrosis. The histology was consistent with pulmonary extramedullary haemopoiesis (EMH). The patient was started on cytoreductive hydroxycarbamide and referred to the local bone marrow transplant unit.

$\mathrm{EMH}$ is the development and growth of haematopoietic tissue outside of the bone marrow. Although essential in foetal life, its occurrence after birth is abnormal. As in physiologic foetal EMH, the liver, spleen and lymph nodes are the most common sites of pathologic EMH, ${ }^{1}$ however,

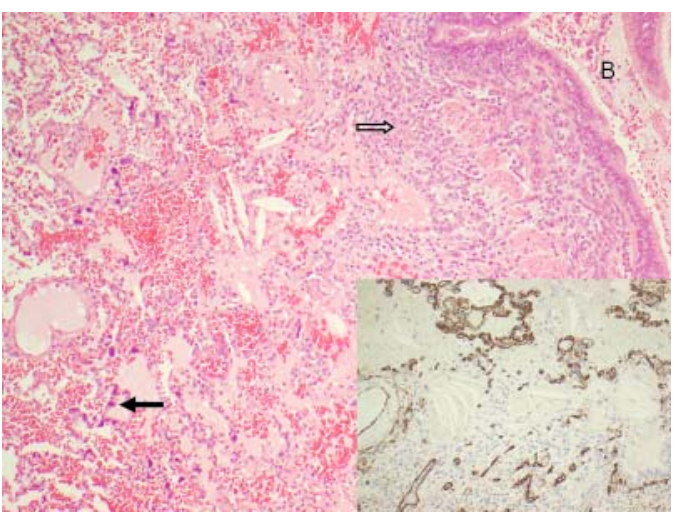

Figure 2 Medium power view of lung with bronchiole (B): Note presence of numerous dark-staining megakaryocytes (solid arrow) within alveolar capillaries and peribronchiolar immature myeloid precursor cells and lymphoid cells (open arrow). Inset-CD34 does stain the capillary endothelium but the not the lymphoid cells, indicating absence of blast cells and effectively excluding leukaemic infiltration. 


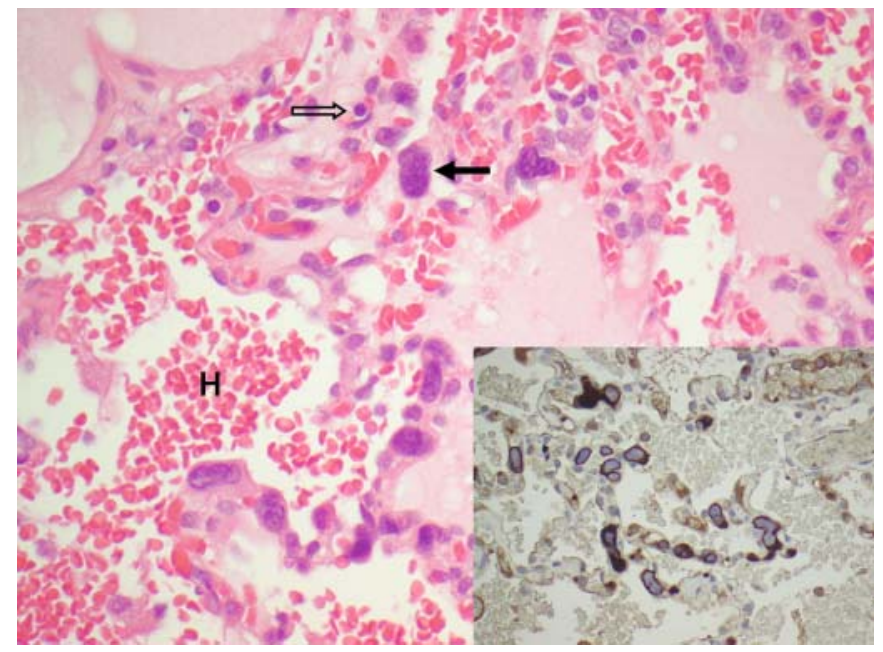

Figure 3 High-power photomicrograph of lung parenchyma: Demonstrates some intra-alveolar haemorrhage $(\mathrm{H})$ and numerous megakaryocytic cells with hyperchromatic, multilobated nuclei (solid arrows), and nucleated erythroid precursors (open arrow). InsetCD42B immunohistochemical stain highlighting megakaryocytes within capillaries $(200 \times)$.

thoracic EMH has been described. ${ }^{2}$ Pleural-based tumours and paraspinal masses are the most common thoracic manifestations of $\mathrm{EMH}^{3}{ }^{3}$ while pulmonary interstitial $\mathrm{EMH}$ is rare and usually occurs in patients with an antecedent haematologic disorder. In suspected pulmonary EMH, surgical lung biopsy is required to allow immunohistochemical staining for erythroid, myeloid and megakaryocyte precursors, ${ }^{4}$ which typically follow the pulmonary lymphatics. The CT findings of pulmonary EMH are nonspecific. However, when this pattern of interlobular septal thickening and centrilobular nodules is encountered in a patient with a haematological malignancy, the diagnosis of pulmonary interstitial EMH should be considered.

\section{REFERENCES}

1 Pitcock JA, Reinhard EH, Justus BW, et al. A clinical and pathological study of seventy cases of myelofibrosis. Ann Intern Med 1962;57:73-84.

2 Koch CA, Li CY, Mesa RA, et al. Nonhepatosplenic extramedullary hematopoiesis: associated diseases, pathology, clinical course, and treatment. Mayo Clinic Proceedings. Mayo Clin Proc 2003;78:1223-33.

3 Lieberman PH, Rosvoll RV, Ley AB. Extramedullary myeloid tumors in primary myelofibrosis. Cancer 1965;18:727-36.

4 Rumi E, Passamonti F, Boveri E, et al. Dyspnea secondary to pulmonary hematopoiesis as presenting symptom of myelofibrosis with myeloid metaplasia. Am J Hematol 2006;81:124-7.

Competing interests None.

Patient consent Obtained.

Provenance and peer review Not commissioned; externally peer reviewed. 